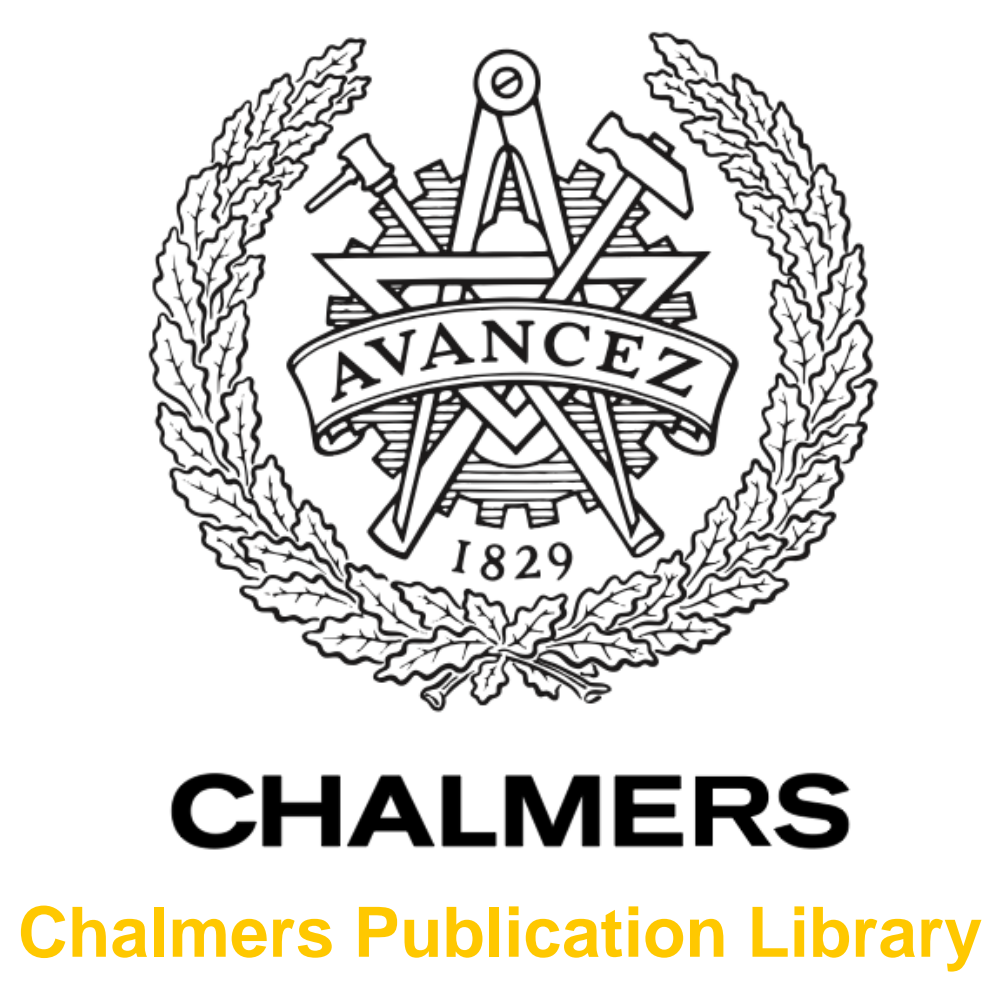

\title{
Mask testing of 28 gbaud 16-QAM transmitters using time-resolved error vector magnitude
}

This document has been downloaded from Chalmers Publication Library (CPL). It is the author's version of a work that was accepted for publication in:

\section{Signal Processing in Photonic Communications, SPPCom 2015; Omni Parker HouseBoston; United States; 27 June 2015 through 1 July 2015}

\author{
Citation for the published paper: \\ Lundström, C. ; Eliasson, H. ; Fatadin, I. et al. (2015) "Mask testing of 28 gbaud 16-QAM \\ transmitters using time-resolved error vector magnitude". Signal Processing in Photonic \\ Communications, SPPCom 2015; Omni Parker HouseBoston; United States; 27 June 2015 \\ through 1 July 2015 pp. SpS4D.3.
}

http://dx.doi.org/10.1364/SPPCOM.2015.SpS4D.3

Downloaded from: http://publications.lib.chalmers.se/publication/222636

Notice: Changes introduced as a result of publishing processes such as copy-editing and formatting may not be reflected in this document. For a definitive version of this work, please refer to the published source. Please note that access to the published version might require a subscription. 


\title{
Mask Testing of 28 Gbaud 16-QAM Transmitters using Time-Resolved Error Vector Magnitude
}

\author{
Carl Lundström ${ }^{1}$, Henrik Eliasson ${ }^{1}$, Irshaad Fatadin $^{2}$, Pontus Johannisson ${ }^{1}$, and Peter A. Andrekson ${ }^{1}$ \\ (1) Photonics Laboratory, Department of Microtechnology and Nanoscience, Chalmers University of Technology, SE-412 96, Gothenburg, Sweden \\ (2) National Physical Laboratory, Teddington, Middlesex, TW11 OLW, U.K. \\ carl.lundstrom@chalmers.se
}

\begin{abstract}
We propose time-resolved EVM for characterization of 16-QAM transmitters. By designing a mask test, different impairments can be separated and quantified. The impact from quadrature error and timing skew are investigated experimentally.

OCIS codes: (060.1660) Coherent communications; (060.4080) Modulation; (060.2330) Fiber optics communications
\end{abstract}

\section{Introduction}

The new generation of coherent fiber-optic transmission systems utilizes modulation formats such as quaternary phase shift keying (QPSK) and 16-ary quadrature amplitude modulation (16-QAM), implying that ways of characterizing and testing transmitters for such formats need to be developed. The root mean square error vector magnitude, $\mathrm{EVM}_{\mathrm{RMS}}$, has been established as a way to quantify performance [1,2]. However, a good evaluation method should account for all possible transmitter errors including incorrect modulator biases, quadrature errors, timing skews etc., and ideally also give information about the origin of the problem and available margins. It should correlate well with the system performance as measured in terms of the bit error rate (BER). An approach based on mask testing of time-resolved $\mathrm{EVM}$, denoted $\mathrm{EVM}_{\mathrm{TR}}$, has been proposed to help address these issues [3]. An $\mathrm{EVM}_{\mathrm{TR}}$ plot is formed by including all samples of the signal and plotting the EVM versus $t$ modulo $T_{s}$, where $T_{s}$ is the symbol duration. Thus, an $\mathrm{EVM}_{\mathrm{TR}}$ plot shows the deviation from the correct constellation point over many superimposed symbol slots.

Traditional mask testing for on-off-keying eye diagrams uses the fact that different transmitter impairments affect the eye in different ways and eventually cause mask hits. The same principle can be applied using $\mathrm{EVM}_{\mathrm{TR}}$, and this approach has been experimentally shown to correlate better than $\mathrm{EVM}_{\mathrm{RMS}}$ to the BER for a polarization-multiplexed (PM) QPSK signal [4]. However, this approach needs to be extended also to larger QAM constellations. In this paper, we apply mask testing based on $\mathrm{EVM}_{\mathrm{TR}}$ to a 16-QAM signal. A transmitter for this format can be implemented in different ways with numerous different potential impairments such as incorrect modulator bias levels, quadrature error (QE) and timing skew, and various gain/amplitude imbalances to name a few. Constructing a mask test method is thus a complex problem. In this initial study we have investigated two common impairments, QE and timing skew that can be considered typical. Additionally, we consider both a parabolic and a polygonal mask shape.

\section{Experimental setup}

The experimental setup is shown in Fig. 1. To generate a 16-QAM signal at 28 Gbaud, a dual-polarization IQ modulator was driven with four binary drive signals to obtain two phase-stable "sub-QPSK" signals in orthogonal polarizations. The timing skew and QEs could be individually adjusted using the delays and phase biases shown in Fig. 1. Using a polarization controller and a linear polarizer (at an angle between the two sub-QPSK signals), these were combined with a $6 \mathrm{~dB}$ power ratio into a single-polarization 16-QAM signal. A tap prior to the polarizer allowed studying the sub-QPSK signals individually. Directly after the transmitter, the signal was split, sending a part into an optical modulation analyzer (OMA, EXFO PSO-200), which measures the signal using equivalent-time sampling and characterizes

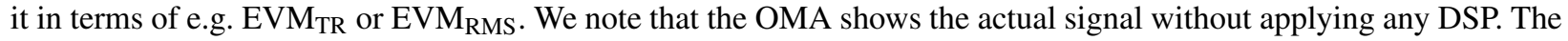
other part of the signal was passed through a noise-loading setup (using a variable optical attenuator and an EDFA) and received with a coherent receiver based on a $100 \mathrm{Gsample} / \mathrm{s}$ real-time sampling oscilloscope with $33 \mathrm{GHz}$ analog bandwidth. The linewidths of both signal and LO lasers were $\sim 100 \mathrm{kHz}$. After sampling, the data was processed in offline DSP. The DSP included IQ angle imbalance compensation based on Gram-Schmidt orthogonalization, resampling to two samples per symbol, decision-directed least-mean square equalization, and frequency offset estimation and carrier phase estimation based on the blind phase search method [5]. Finally, the BER was evaluated by direct error counting. 


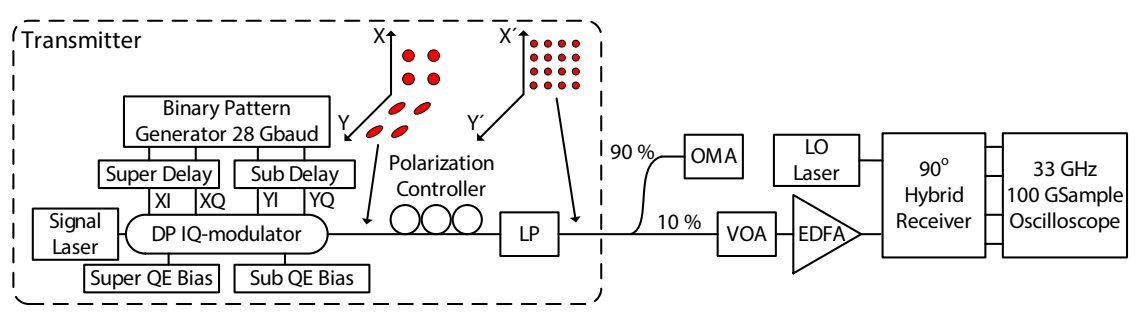

Fig. 1. The experimental setup showing how a PM-QPSK signal is converted into a singlepolarization 16-QAM. PC: Polarization controller. LP: Linear polarizer. OMA: Optical modulation analyzer. VOA: Variable optical attenuator.

\section{Experimental results and discussion}

To quantify the effects of the transmitter impairments, QE and timing skew were introduced separately into the two sub-QPSK signals, which we, refer to as the large and small sub-QPSK, respectively. The OSNR penalty at a BER of $10^{-3}$ was measured for various levels of each impairment. An $\mathrm{EVM}_{\mathrm{TR}}$ plot was also saved for mask testing. The OSNR penalty is shown in Fig. 2, with $1 \mathrm{~dB}$ penalty marked in the figures. As expected, induced QE on the large sub-QPSK yields a larger penalty than induced QE on the small sub-QPSK. The QE that yields about $1 \mathrm{~dB}$ OSNR penalty is 5 degrees and 17.5 degrees, respectively. Timing skew, however, yields similar, and relatively low penalties for the values tested, with a skew of about 25 percent of the symbol slot required in both cases to incur an OSNR penalty of more than $1 \mathrm{~dB}$.

Fig. 3 shows $\mathrm{EVM}_{\mathrm{TR}}$ as measured directly after the transmitter with induced impairments giving approximately a $1 \mathrm{~dB}$ OSNR penalty. The $\mathrm{EVM}_{\mathrm{TR}}$ for the ideal case without induced impairment is also shown. Previous works [3,4] have not included any discussion on mask design, and only suggested potential mask shapes. A suitable mask should be equidistant from the $\mathrm{EVM}_{\mathrm{TR}}$-rails for an ideal signal, and show similar performance for all potential transmitter impairments. At the same time it is desirable that it is not too complex to define. The two impairments considered here, $\mathrm{QE}$ and skew, qualitatively affect the $\mathrm{EVM}_{\mathrm{TR}}$ in two different ways, $\mathrm{QE}$ tends to push the bottom rail up towards higher EVM values, and skew tends to push the side rails toward the center of the symbol slot. Here, we have investigated a simple mask construction, namely a parabola with an offset vertex above the zero EVM line, giving only two free parameters. By optimizing the parabola, with respect to the variance of the number of mask hits for the different impairments studied the best mask choice can be found. We additionally require the mask to give at least three times as many hits for any impairment giving $1 \mathrm{~dB}$ penalty than for the ideal case and also do not consider samples in the top of the sampling window (normalized EVM > 0.7). Another possible mask design is a polygon. A polygon with six vertices (two isoceles trapezoids) that closely approximated the previously found parabola gave slightly more consistent performance among the different impairments. This is attributed to the polygonal mask being slightly more

(a)

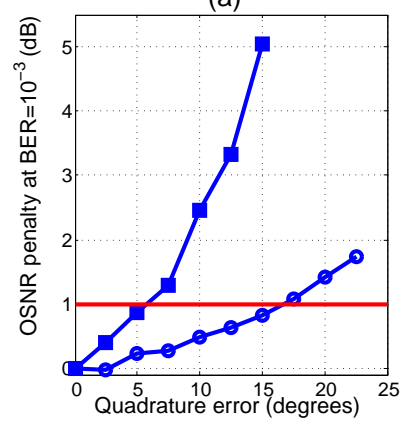

(b)

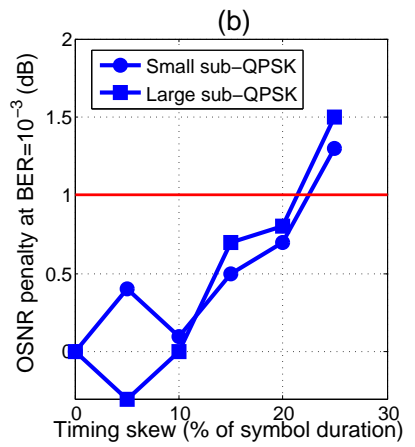

Fig. 2. OSNR penalties at BER $=10^{-3}$ for varying amounts of (a) $\mathrm{QE}$ and (b) timing skew on the two sub-QPSKs, respectively. 

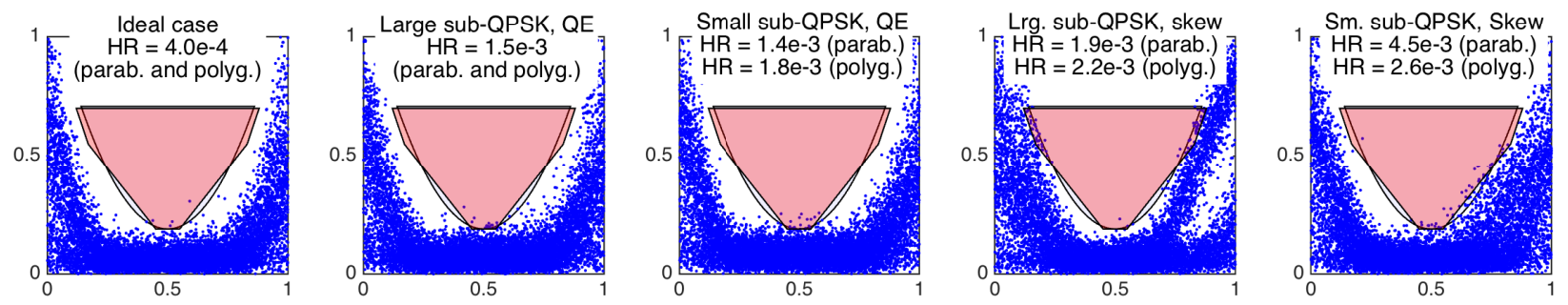

Fig. 3. Time-resolved EVM as a function of time within the symbol slot. The impact of QE and skew on the large and small sub-QPSK signals, respectively, is quantified in terms of the mask hit ratio (HR). The OSNR penalty was close to $1 \mathrm{~dB}$ in all cases except the ideal case, which is included as a reference. Two mask shapes, parabolic and polygonal, are shown.

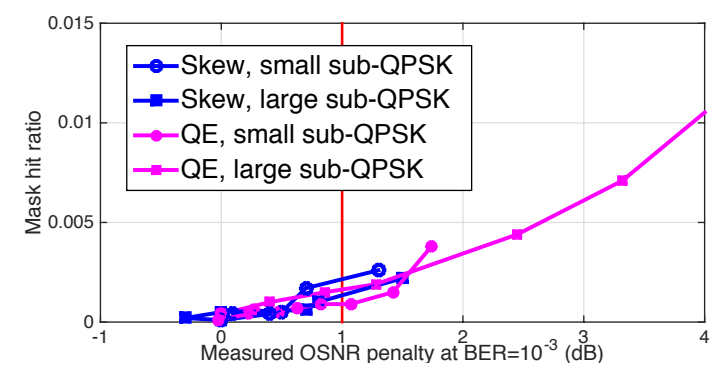

Fig. 4. The number of mask hits for the different impairments versus OSNR penalty.

narrow in its lower part, reducing the number of mask hits for timing skew on the small sub-QPSK. Fig. 3 includes both of the proposed mask shapes and indicate the hit ratios.

In Fig. 4 the number of mask hits (polygon-shaped mask) is plotted versus the measured OSNR penalty for the different impairments. The mask hits follow a similar curve, for all impairments though there is a noticeable measurement uncertainty due to the noise of the signal. Clearly, it is possible to find a choice of mask that yields a similar number of mask hits for all impairments, while the number of hits for the ideal case is significantly lower.

\section{Conclusion}

We have proposed and used time-resolved EVM measurements for 16-QAM by studying the impact of quadrature error and timing skew in a dual-parallel IQ modulator 16-QAM transmitter in terms of OSNR penalty, EVM $_{\text {RMS }}$, and $\mathrm{EVM}_{\mathrm{TR}}$-mask hits. It was found that with a suitable choice of mask the number of mask hits can correlate well for the impairments studied. Further investigations will include more impairments. The performance is very sensitive to the mask choice and this needs to be carefully studied. Mask tests similar to the proposed ones are candidates for standardized transmitter testing.

This work was supported by EMRP IND51 "MORSE". The EMRP is jointly funded by the EMRP participating countries within Euramet and the European Union. EXFO is acknowledged for the loan of the OMA.

\section{References}

[1] R. A. Shafik, M. S. Rahman, and A. R. Islam, "On the extended relationships among EVM, BER and SNR as performance metrics," in "International Conference on Electrical and Computer Engineering (ICECE)," (2006), pp. 408-411.

[2] R. Schmogrow, B. Nebendahl, M. Winter, A. Josten, D. Hillerkuss, S. Koenig, J. Meyer, M. Dreschmann, M. Huebner, C. Koos, J. Becker, W. Freude, and J. Leuthold, "Error vector magnitude as a performance measure for advanced modulation formats," IEEE Photon. Technol. Lett. 24, 61-63 (2012).

[3] H. Sunnerud, M. Sköld, M. Westlund, and P. A. Andrekson, "Characterization of complex optical modulation formats at $100 \mathrm{~Gb} / \mathrm{s}$ and beyond by coherent optical sampling," J. Lightw. Technol. 30, 3747-3759 (2012).

[4] H. Eliasson, P. Johannisson, H. Sunnerud, M. Westlund, M. Karlsson, and P. A. Andrekson, "Transmitter mask testing for 28 GBaud PM-QPSK," in "European Conference on Optical Communication (ECOC)," (2013), p. Tu.3.C.2.

[5] T. Pfau, S. Hoffmann, and R. Noé, "Hardware-efficient coherent digital receiver concept with feedforward carrier recovery for M-QAM constellations," J. Lightw. Technol. 27, 989-999 (2009). 